\title{
O impacto da tecnologia no tratamento do trauma vascular iatrogênico cervicotorácico: estudo de dois casos com intervalo de três décadas e revisão da literatura
}

\author{
The impact of technology on treatment of iatrogenic cervicothoracic vascular traumas: \\ a study of two cases three decades apart and a review of the literature
}

Victor Bilman ${ }^{1,2}$, Bernardo Massière $^{1,2}$, Alberto Vescovi ${ }^{1,2}$, Daniel Leal ${ }^{1,2}$, Paula Vivas ${ }^{1,2}$, Bruno Demier $^{1,2}$, Arno von Ristow ${ }^{1,2,3}$

\begin{abstract}
Resumo
Complicações relacionadas ao acesso venoso cervicotorácico, como os pseudoaneurismas (PAs), podem ser devastadoras. Neste artigo, apresentamos dois casos semelhantes em que o avanço tecnológico impactou no diagnóstico, tratamento e resultados. Ambos pacientes apresentaram volumoso PA após a tentativa de punção venosa profunda. O primeiro caso, em 1993, diagnosticado por duplex scan, revelou grande PA oriundo da artéria subclávia direita. A artéria foi abordada por esternotomia mediana com extensão supraclavicular. O PA originava-se do tronco tireocervical, tratado com simples ligadura. No segundo caso, em 2017, angiotomografia revelou um PA originário da artéria vertebral, que foi tratado com técnica endovascular, mantendo a perviedade do vaso. Ambos evoluíram satisfatoriamente, apesar de abordagens bastante diferentes. A lesão vascular cervicotorácica representa um desafio propedêutico e terapêutico, com alto risco de ruptura. Os avanços tecnológicos diminuem os riscos de lesões vasculares com acesso cirúrgico difícil e devem estar entre as opções do cirurgião vascular.
\end{abstract}

Palavras-chave: falso aneurisma; artéria vertebral; cateterismo venoso central; procedimentos endovasculares; stents metálicos autoexpansíveis.

\begin{abstract}
Complications such as pseudoaneurysms (PA) related to cervicothoracic venous access can be devastating. In this article, we present two similar cases in which technological advances impacted diagnosis, treatment, and results. Both patients developed massive PA after deep venous puncture attempts. The first case occurred in 1993 and was diagnosed by a duplex scan that revealed a large PA originating from the right subclavian artery. The artery was approached by median sternotomy with supraclavicular extension. The PA originated from the thyrocervical trunk and was treated with simple ligation. The second case was in 2017. Angiotomography revealed a PA originating in the vertebral artery, which was treated with endovascular techniques, maintaining vessel patency. Both patients progressed satisfactorily, despite quite different approaches. Cervicothoracic vascular lesions represent a diagnostic and therapeutic challenge, where the risk of rupture is high. Technological advances have reduced the risks involved in management of vascular injuries with difficult surgical access.
\end{abstract}

Keywords: Aneurysm, false; vertebral artery; central venous access; endovascular procedures; self-expanding metallic stents.

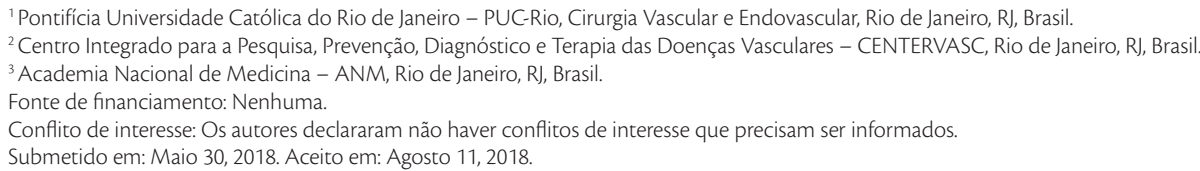




\section{INTRODUÇÃO}

Os acessos venosos profundos tornaram-se prática rotineira nos hospitais. Seu uso é essencial para procedimentos diagnósticos e terapêuticos ${ }^{1-3}$. A lesão iatrogênica da artéria subclávia e seus ramos é uma complicação rara, mas associada a grave morbidade e mortalidade ${ }^{4,5}$. Inamasu et al. e Bernik et al. relatam incidência de lesões arteriais pós-punção variando de 0,4 a $9,9 \%$ e 0,5 a $11,4 \%$, respectivamente, mais comumente associadas à artéria carótida comum. Os sintomas se apresentam, na maioria dos casos, de forma tardia, sendo os principais as fístulas arteriovenosas e os pseudoaneurismas (PAs) ${ }^{1,3}$.

O manejo dessas lesões iatrogênicas apresentou grande avanço com o advento da cirurgia endovascular. Apesar de Inamasu et al. descreverem a excisão cirúrgica do PA com ou sem reconstrução arterial como principal tratamento, o complexo acesso cirúrgico pode levar a várias complicações ${ }^{1}$.

O presente artigo é um estudo comparativo de dois casos de lesões iatrogênicas semelhantes, pós-punção de acesso venoso profundo, mas com diagnósticos e abordagens diferentes. O objetivo é demonstrar que a evolução tecnológica impactou no tratamento do trauma vascular cervicotorácico de difícil acesso. Uma detalhada revisão da literatura contribui para o estudo da evolução desse tema e corrobora nossas conclusões.

\section{DESCRIÇÃO DOS CASOS}

O primeiro caso, ocorrido em setembro de 1993, refere-se a uma paciente de 72 anos de idade, sexo feminino, portadora de cardiopatia grave, hipertensão arterial e obesidade grau III, internada em unidade de terapia intensiva com sepse de origem pulmonar. Foi submetida a punção de veia subclávia direita por técnica supraclavicular, sem sucesso. Uma semana depois, foi solicitado parecer à equipe de cirurgia vascular, sendo observada volumosa massa pulsátil na região cervical direita. Ao exame físico, a paciente apresentava-se hemodinamicamente instável, com uma volumosa massa pulsátil cervical direita (Figura 1). O exame de duplex scan demonstrou a presença de PA oriundo da segunda porção da artéria subclávia direita (ASD), com $50 \times 42 \mathrm{~mm}$ de diâmetro (Figura 2), sendo indicada cirurgia direta.

A esternotomia mediana foi o acesso realizado, com extensão supraclavicular direita (Figura 3). Procedeu-se ao isolamento da ASD proximal e progressivamente foi abordado o colo do PA. Este era originário do segmento proximal do tronco tireocervical, que foi simplesmente ligado, e o saco aneurismático foi esvaziado. A evolução foi satisfatória, apesar da magnitude da intervenção e da gravidade da paciente.

O segundo caso, ocorrido em dezembro de 2017, refere-se a uma paciente de 66 anos de idade, sexo feminino, portadora de cardiopatia grave e hipertensão arterial sistêmica, e submetida a transplante renal em 2007 devido a doença renal policística. Foi internada em unidade de terapia intensiva com sepse de origem pulmonar. Foi submetida a tentativa de punção de veia jugular interna esquerda ecoguiada para administração de aminas vasoativas; sem sucesso, o procedimento foi abandonado. A paciente evoluiu com massa pulsátil na região cervical esquerda, associada a queda progressiva do hematócrito. Após 15 dias de evolução com dor e expansão da massa cervical, foi solicitada avaliação da cirurgia vascular.

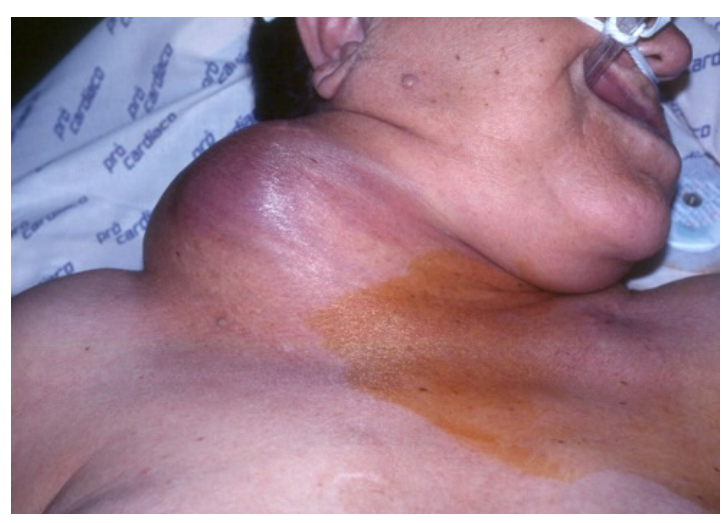

Figura 1. Massa cervical esquerda associada a hematoma.

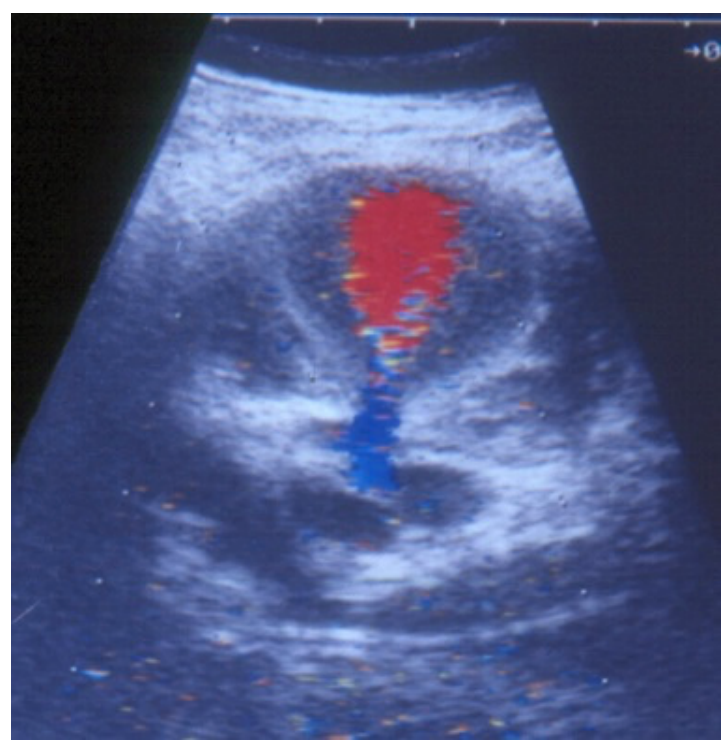

Figura 2. Eco-Doppler demonstrando pseudoaneurisma com fluxo turbulento. 
Ao exame físico e anamnese, a paciente queixou-se de dor importante na região cervical esquerda, associada a massa pulsátil (Figura 4). $\mathrm{O}$ duplex scan sugeriu a presença de PA oriundo da artéria carótida comum esquerda (Figura 5), e a angiotomografia computadorizada (ATC) demonstrou a presença de PA do segmento V1 da artéria vertebral esquerda, com 30 × 32 mm de diâmetro (Figura 6).

Após discussão em equipe, optou-se pelo tratamento endovascular, com implante de um stent revestido Viabahn ${ }^{\circledR} 5 \mathrm{~mm} \times 2,5 \mathrm{~cm}$ (WL Gore, Flagstaff, AZ, EUA) na artéria vertebral. O procedimento transcorreu sem intercorrências, sendo utilizados $18 \mathrm{~mL}$ de contraste iodado (Figura 7). A paciente evoluiu com regressão da massa cervical e resolução da dor no

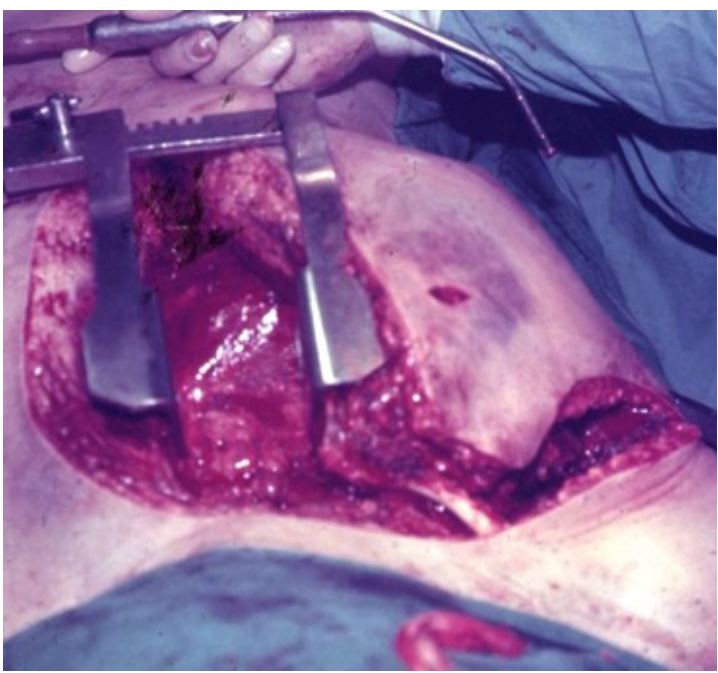

Figura 3. Esternotomia com extensão supraclavicular direita.

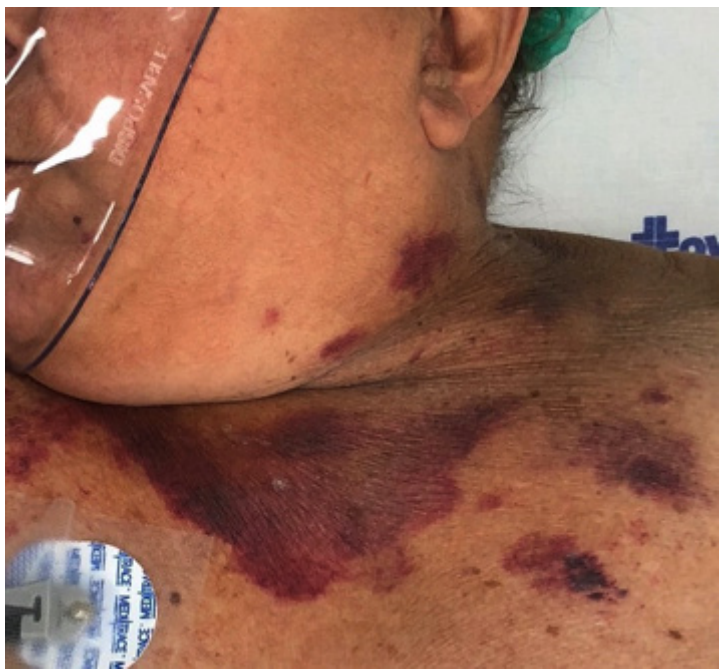

Figura 4. Massa cervical esquerda associada a hematoma. pós-operatório imediato, sem déficit neurológico. Duplex scan de controle realizado após 6 meses do procedimento demonstrou perviedade da artéria vertebral esquerda ao nível do segmento V3.

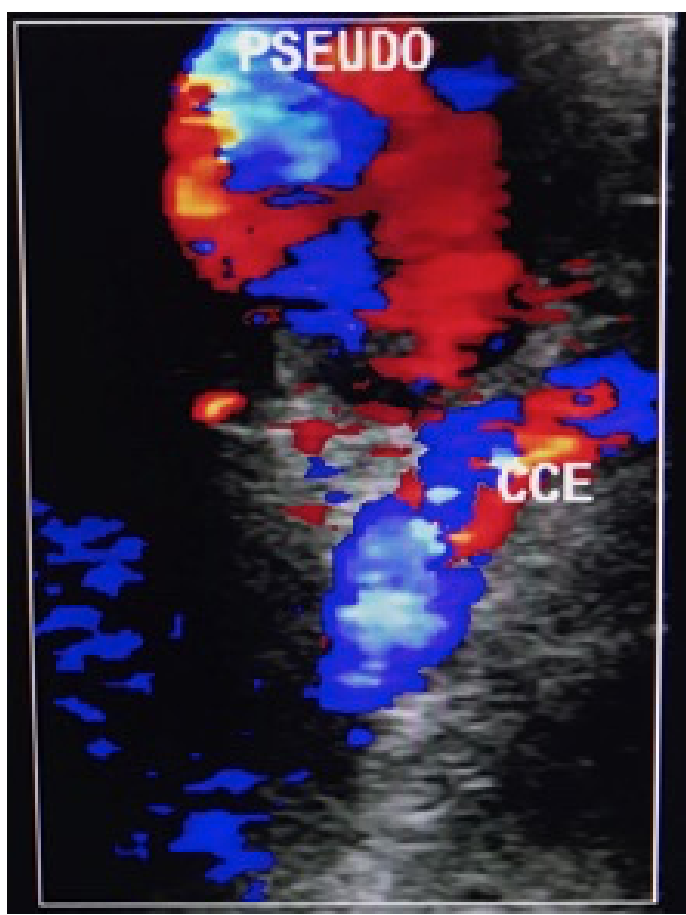

Figura 5. Eco-Doppler demonstrando pseudoaneurisma (origem suspeitada ao nível da carótida comum esquerda - CCE), com fluxo turbulento.

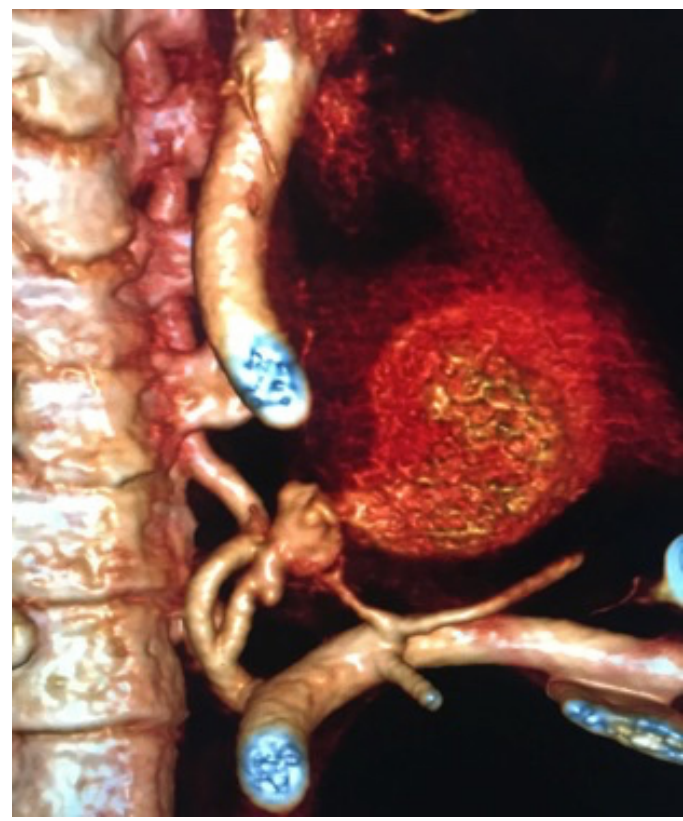

Figura 6. Reconstrução 3D de angiotomografia da artéria vertebral esquerda, com o enchimento do pseudoaneurisma. 


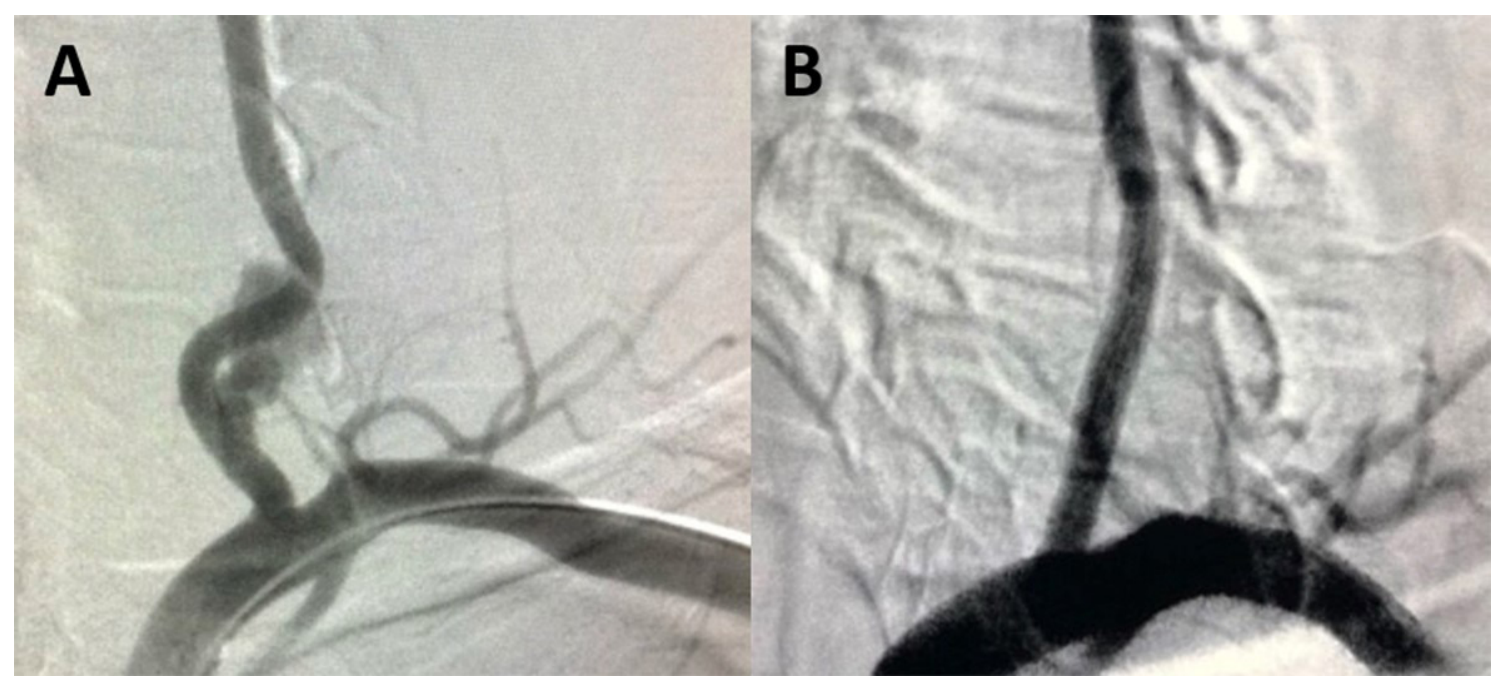

Figura 7. (A) Arteriografia peroperatória vertebral esquerda; (B) Arteriografia de controle pós-implante de Viabahn ${ }^{\circledR} 5 \mathrm{~mm} \times 2,5 \mathrm{~cm}$ (WL Gore, Flagstaff, AZ, EUA).

\section{DISCUSSÃO}

As complicações vasculares relacionadas a procedimentos percutâneos na região cervicotorácica podem ser devastadoras ${ }^{4}$. Trauma iatrogênico em artérias carótidas e subclávias pode provocar sangramento intenso, hematomas, dissecção, embolia ou trombose, sendo descritos na literatura médica casos de obstrução de vias aéreas secundária a hematoma cervical em expansão, choque por hemotórax, acidente vascular cerebral devido a embolia, PA e fístula arteriovenosa ${ }^{4}$. O PA provavelmente é a complicação mais rara de lesão em artéria vertebral, com poucos casos descritos na literatura ${ }^{3,5-8}$ e incidência de $0,2 \%$, conforme reportado por Elias et al. ${ }^{2}$.

Os PAs da artéria vertebral podem ocorrer devido a trauma penetrante ou contuso da região cervical, dissecção arterial e após procedimento cirúrgico. Lesões penetrantes envolvem principalmente a primeira porção da artéria, antes da entrada pelo forame transverso, enquanto traumas contusos cervicais e cranianos acometem as porções mais distais ${ }^{2,7}$. A rápida identificação e conduta dessas lesões é primordial para um resultado satisfatório, apesar de o manejo ser pouco compreendido e raramente descrito na literatura $^{1,2}$. Embora a resolução espontânea desses PAs ter sido descrita, rotura é observada em 31 a 54\% dos pacientes ${ }^{4,9}$.

Os PAs se apresentam, na maioria dos casos, de forma tardia e principalmente sob a forma de massa pulsátil, com dor local, dispneia, insuficiência cardíaca e isquemia cerebral e medular ${ }^{1-3}$. Ambekar et al. descrevem diagnóstico após dois dias, Elias et al., após três dias,
Bernik et al., após quatro dias, Amaral et al., após 15 dias, e Cihangiroglu, após 32 dias $^{2,3,5,7,10}$.

A abordagem diagnóstica inicial descrita na literatura foi a realização de duplex scan, com visualização de hematoma com fluxo arterial em seu interior. Apesar de ser um bom método, a origem do PA pode não ser muito bem visualizada, conforme afirmam Cihangiroglu et al. ${ }^{3,7}$. A proximidade com a artéria carótida interna, o volume do hematoma e a turgescência da veia jugular interna são fatores que dificultam a visibilização de sua origem. Isso aconteceu nos dois casos relatados neste estudo. A ATC demonstra superioridade na visualização da lesão, incluindo sua origem, correta localização, presença de traumas vasculares adicionais e perviedade da artéria vertebral contralateral, além de auxiliar no planejamento terapêutico ${ }^{2,3,7}$.

O tratamento de PA inclui embolização endovascular, reparo direto da artéria vertebral ou ressecção do PA com ligadura ${ }^{2,7}$. Bernik et al. ainda inclui compressão guiada por ultrassom e injeção percutânea de trombina como opções alternativas ${ }^{3}$. Vale ressaltar que a profundidade da lesão impossibilita o tratamento compressivo e, pelo risco de embolização cerebral, o tratamento com trombina não é recomendado ${ }^{3}$. Aoki et al. descrevem o caso de um paciente tratado com conduta conservadora e que, após o $70^{\circ}$ dia, evoluiu com dor e parestesia do ombro e braço direitos devido a compressão do plexo braquial pelo hematoma. $\mathrm{O}$ paciente evoluiu com rotura do $\mathrm{PA}$, sendo operado em caráter de emergência com reparo direto; porém, foi a óbito no $11^{\mathrm{o}}$ dia de pós-operatório ${ }^{8}$.

Amaral et al. sugere o tratamento aberto como a primeira opção terapêutica quando a lesão é 
sintomática e localizada na origem da artéria vertebral ${ }^{10}$. A esternotomia mediana com extensão supra ou transclavicular é a melhor opção para controle proximal em vigência de grande hematoma à direita ${ }^{3,7,10}$. Outra opção de acesso é uma única incisão ao longo do músculo esternocleidomastóideo ou transclavicular, combinada com implante de balão temporário na origem da artéria vertebral ${ }^{10}$. Bernik et al. enfatizam a necessidade de um controle proximal ótimo para melhor visualização da lesão e tratamento cirúrgico ${ }^{3}$. À esquerda, uma abordagem envolveria toracotomia anterior para controle proximal da subclávia, pois a localização posterior dessa artéria não permite bom controle por esternotomia ${ }^{11}$. Todas essas abordagens envolvem morbidade elevada ${ }^{11,12}$.

$\mathrm{Na}$ literatura, o tratamento endovascular tem apresentado bons resultados ${ }^{4,13-16}$. Essa abordagem configurou uma mudança no manejo da lesão, que antes era realizado frequentemente por ligadura, para estratégias de exclusão do PA, mantendo a perviedade do vaso. Ambekar et al. e Kerolus et al. descrevem o uso de dispositivos de embolização como o Pipeline ${ }^{\circledR}$ (Covidien Vascular Therapies, Mansfield, MA, EUA), que promovem desvio de fluxo preservando a perviedade dos vasos. Em uma revisão sistemática, obtiveram uma taxa de obliteração aneurismática de $82,9 \%$ em 6 meses $^{5}$. Pérez et al. utilizaram stent de cromo-cobalto balão-expansível revestido (Papyrus PK Biotronik ${ }^{\circledR}$ ) para a exclusão de um PA da artéria vertebral, obtendo sucesso e mantendo a perviedade do vaso $^{13}$. Kwon et al., além do stent revestido, também embolizaram o PA utilizando molas e obtiveram sucesso $^{16}$. Yamamoto et al. descrevem o uso de molas e N-butil-2-cianoacrilato, com consequente oclusão da lesão, mas também da artéria vertebral ${ }^{17}$. Relatamos, provavelmente pela primeira vez, o uso de um stent revestido Viabahn ${ }^{\circledR} 5 \mathrm{~mm} \times 2,5 \mathrm{~cm}$ (WL Gore, Flagstaff, AZ, EUA) na artéria vertebral, mantendo o vaso pérvio.

Assim como descrito por Amaral et al. e Yamamato et al., a oclusão unilateral da artéria vertebral é uma prática comum para o tratamento das lesões e, quando há perviedade da artéria contralateral, geralmente é bem tolerada ${ }^{10,17}$. A artéria cerebelar posteroinferior no lado da lesão deve ser suprida pela vertebral contralateral; se isso não ocorrer, pode-se desenvolver a síndrome de Wallenberg ${ }^{10}$. Essa síndrome é caracterizada por déficit nos nervos cranianos (V, IX, X e XI), ataxia, síndrome de Horner e perda contralateral da sensação de dor e temperatura, entre outras manifestações de insuficiência vertebrobasilar. É importante destacar que $15 \%$ da população tem uma artéria vertebral dominante, sendo a outra hipoplásica, e que $5 \%$ tem apenas uma artéria vertebral ${ }^{10}$. A oclusão ou ligadura da artéria vertebral em situações não emergenciais requer confirmação de fluxo sanguíneo adequado para a circulação posterior ${ }^{10,18,19}$.

\section{CONCLUSÃO}

Embora usuais na medicina moderna, as punções venosas profundas não estão livres de complicações. O tratamento endovascular tem um importante papel na abordagem de pacientes com trauma vascular iatrogênico. Com isso, o papel do cirurgião vascular tradicional tem sido desafiado. Assim como na técnica de reparo aberto, o cirurgião vascular deve estar habituado com os avanços tecnológicos, tanto na propedêutica quanto na terapêutica. Com a técnica endovascular, geralmente há uma diminuição da morbidade e mortalidade no manejo de lesões de difícil acesso.

A introdução de novas tecnologias, como a punção ecoguiada, apesar de diminuir a ocorrência de complicações do procedimento, não evita iatrogenias. Apesar de treinamento e experiência, as punções venosas não estão livres de riscos. O conhecimento da anatomia cervical, bem como das suas variações anatômicas, faz-se necessário de modo tão relevante quanto o uso judicioso da ultrassonografia. A prevenção de lesões iatrogênicas é o principal ponto a ser ensinado nas instituições hospitalares ${ }^{6}$.

\section{REFERÊNCIAS}

1. Inamasu J, Guiot $\mathrm{BH}$. latrogenic vertebral artery injury. Acta Neurol Scand. 2005;112(6):349-57. http://dx.doi.org/10.1111/j.16000404.2005.00497.x. PMid:16281916.

2. Elias M. Pulsating mass in the neck following attempted internal jugular vein catheterisation. Anaesthesia. 1999;54(9):914-5. http:// dx.doi.org/10.1046/j.1365-2044.1999.01089.x. PMid:10460719.

3. Bernik TR, Friedman SG, Scher LA, Safa T. Pseudoaneurysm of the subclavian-vertebral artery junction - case report and review of the literature. Vasc Endovascular Surg. 2002;36(6):461-4. http:// dx.doi.org/10.1177/153857440203600607. PMid:12476236.

4. Guilbert MC, Elkouri S, Bracco D, et al. Arterial trauma during central venous catheter insertion: case series, review and proposed algorithm. J Vasc Surg. 2008;48(4):918-25. http://dx.doi.org/10.1016/j. jvs.2008.04.046. PMid:18703308.

5. Ambekar S, Sharma M, Smith D, Cuellar H. Successful treatment of iatrogenic vertebral pseudoaneurysm using pipeline embolization device. Case Rep Vasc Med. 2014;2014:341748. http://dx.doi. org/10.1155/2014/341748. PMid:25276469.

6. Yu NR, Eberhardt RT, Menzoian JO, Urick CL, Raffetto JD. Vertebral artery dissection following intravascular catheter placement: a case report and review of the literature. Vasc Med. 2004;9(3):199-203. http://dx.doi.org/10.1191/1358863x04vm565cr. PMid:15675185.

7. Cihangiroglu $M$, Rahman A, Yildirim $H$, Burma $O$, Uysal $H$. latrogenic vertebral artery pseudoaneurysm: US, CT and MRI findings. Eur J Radiol. 2002;43(1):14-8. http://dx.doi.org/10.1016/ S0720-048X(01)00451-X. PMid:12065115. 
8. Aoki H, Mizobe T, Nozuchi S, Hatanaka T, Tanaka Y. Vertebral artery pseudoaneurysm: a rare complication of internal jugular vein catheterization. Anesth Analg. 1992;75(2):296-8. http://dx.doi. org/10.1213/00000539-199208000-00027. PMid:1632547.

9. Yilmaz MB, Donmez H, Tonge M, Senol S, Tekiner A. Vertebrojugular arteriovenous fistula and pseudoaneurysm formation due to penetrating vertebralartery injury: case report and review of the literature. Turk Neurosurg. 2015;25(1):141-5. PMid:25640560.

10. Amaral JF, Grigoriev VE, Dorfman GS, Carney WI Jr. Vertebral artery pseudoaneurysm. A rare complication of subclavian artery catheterization. Arch Surg. 1990;125(4):546-7. http://dx.doi. org/10.1001/archsurg.1990.01410160134026. PMid:2322123.

11. Perissé RSP, Ristow AV, Palazzo JCC. Traumatismos vasculares da região cérvico-torácica e cervical. In: Ristow AV, Moreira RSP, editors. Urgências vasculares. Rio de Janeiro: Editora Cultura Médica; 1983. p. 76-101.

12. Meirelles SSL, Ristow AV. Trauma dos vasos cervicais. In: Freire E, editor. Trauma. Rio de Janeiro: Atheneu; 2001. p. 1341-1364.

13. Pérez $\mathrm{GCl}$, Morata AR, Ortega JPR, Medialdea RG, García PC. Endovascular Treatment of a Symptomatic Vertebral Artery Pseudoaneurysm. Ann Vasc Surg. 2015;29(5):1018.e5-8. http:// dx.doi.org/10.1016/j.avsg.2015.01.022. PMid:25770383.

14. Guan Q, Chen L, Long Y, Xiang Z. latrogenic vertebral artery injury during anterior cervical spine surgery. A systematic review. World Neurosurg. 2017;106:715-22. http://dx.doi.org/10.1016/j. wneu.2017.07.027. PMid:28712898.

15. Kerolus M, Tan LA, Chen M. Treatment of a giant vertebral artery pseudoaneurysm secondary to gunshot wound to the neck using pipeline embolization device. Br J Neurosurg. 2016;1-2. http:// dx.doi.org/10.1080/02688697.2016.1265087. PMid:27927019.

16. Kwon SH, Oh JH. A large vertebral artery pseudoaneurysm due to percutaneous internal jugular vein cannulation. Intensive Care Med. 2014;40(12):1934. http://dx.doi.org/10.1007/s00134-0143441-2. PMid:25118869.

17. Yamamoto A, Suzuki K, Sakaida H, Suzuki H, Imai H. Management of inadvertent vertebral artery injury due to central venous catheterization in a coagulopathic patient. Acute Med Surg. 2016;3(3):265-7. http://dx.doi.org/10.1002/ams2.177. PMid:29123795.

18. Aydin E, Gok M, Esenkaya A, Cinar C, Oran I. Endovascular Management of latrogenic Vascular Injury in the Craniocervical Region. Turk Neurosurg. 2018;28(1):72-8. PMid:27593845.
19. Abeysinghe $\mathrm{V}, \mathrm{Xu} \mathrm{JH}$, Sieunarine K. latrogenic injury of vertebral artery resulting in stroke after central venous line insertion. BMJ Case Rep. 2017;2017. http://dx.doi.org/10.1136/bcr-2017-222429. PMid:29167220.

Correspondência Victor Bilman

Centro Integrado para a Pesquisa, Prevenção, Diagnóstico e Terapia das Doenças Vasculares - Centervasc Rua Sorocaba, $477,8^{\circ}$ andar, Botafogo CEP 22271-110 - Rio de Janeiro (RJ), Brasil Tel.: (21) 2226.5858 E-mail:vbilman@gmail.com

Informações sobre os autores

VB e BD - Pós-graduandos em Cirurgia Vascular e Endovascular, Centro Integrado para a Pesquisa, Prevenção, Diagnóstico e Terapia das Doenças Vasculares (CENTERVASC Rio), Pontifícia Universidade Católica do Rio de Janeiro (PUC-Rio)

BM, AV, DL e PV- Cirurgiões vasculares, chefes, Serviço de Cirurgia Vascular e Endovascular, Centro Integrado para a Pesquisa, Prevenção,

Diagnóstico e Terapia das Doenças Vasculares (CENTERVASC Rio),

Pontifícia Universidade Católica do Rio de Janeiro (PUC-Rio)

AVR - Cirurgião vascular e coordenador chefe, Serviço de Cirurgia Vascular e Endovascular, Pontifícia Universidade Católica do Rio de Janeiro (PUC-Rio); Diretor científico, Centro Integrado para a Pesquisa, Prevenção, Diagnóstico e Terapia das Doenças Vasculares,Pontifícia Universidade Católica do Rio de Janeiro (CENTERVASC Rio - PUC Rio); Acadêmico da Academia Nacional de Medicina (ANM).

Contribuições dos autores Concepção e desenho do estudo: AVR, VB, BM Análise e interpretação dos dados: $A V R, V B, B D$

Coleta de dados: VB, BM, PV Redação do artigo: AVR, VB Revisão crítica do texto: AVR, AV, DL, PV, BD Aprovação final do artigo*: AVR Análise estatística: N/A Responsabilidade geral pelo estudo: AVR

*Todos os autores leram e aprovaram a versão final submetida ao I Vasc Bras. 